\title{
Facilitating Lithium-Ion Conduction in Gel Polymer Electrolyte by Metal-Organic Frameworks
}

\author{
Xing Lu, ${ }^{\mathrm{a} \dagger}$ Haiping Wu,${ }^{\mathrm{a} \dagger}$ Dejia Kong, ${ }^{\mathrm{a}}$ Xinru Li, ${ }^{\mathrm{a}}$ Li Shen, ${ }^{* \mathrm{a}}$ and Yunfeng $\mathrm{Lu}^{* \mathrm{a}}$ \\ a. Department of Chemical and Biomolecular Engineering, University of California, Los Angeles, \\ CA 90095, United States
}

\section{Experimental section}

Synthesis of UiO-66. Terephthalic acid (10 mmol, $1.66 \mathrm{~g})$ was dissolved in DMF $180 \mathrm{~mL}$, benzoic acid (10 equivalents with respect to $\left.\mathrm{ZrCl}_{4}\right)$, and concentrated $\mathrm{HCl}(1.65 \mathrm{~mL})$ were added into the solution. The solution was stirred at room temperature for $0.5 \mathrm{~h}$, then $\mathrm{ZrCl}_{4}(10 \mathrm{mmol}, 2.33 \mathrm{~g})$ was dissolved in the solution. The reaction vessel was transferred to an oven and heated at $120{ }^{\circ} \mathrm{C}$ for $72 \mathrm{~h}$. The solution was cooled to room temperature, and the precipitates were isolated by centrifugation. The solids were suspended in DMF and stirred overnight. The suspension was centrifuged, and the solid was washed with DMF for five times. The obtained solid was washed with methanol in the same way as described for washing with DMF. Finally, the solid was dried at $60{ }^{\circ} \mathrm{C}$ overnight under vacuum.

Preparation of gel polymer electrolytes. The preparation of MGPL-12 was exemplified herein. The UiO-66 particles were initially activated at $300{ }^{\circ} \mathrm{C}$ for $24 \mathrm{~h}$ and then transferred to the glove box (Argon atmosphere). $63 \mathrm{mg}$ of UiO-66 particles were immersed in $1 \mathrm{M} \mathrm{LiClO}_{4}$ in $\mathrm{PC}(1.2 \mathrm{~mL})$ electrolyte overnight, then $6 \mathrm{~mL}$ dimethyl carbonate (DMC) was added. The suspension was stirred for $6 \mathrm{~h}$ before poly(vinylidene fluoride-co-hexafluoropropylene) (PVDF-HFP) (460 mg) was added into the suspension. The mixture was stirred at $60^{\circ} \mathrm{C}$ overnight. $1.5 \mathrm{~mL}$ suspension was cast on a polypropylene (PP) plate. After evaporation of the solvents, the resulting gel polymer electrolyte could be peeled off as a self-standing membrane. Pure gel polymer electrolyte was prepared similarly without the addition of $\mathrm{UiO}-66$. The plasticizer $(\mathrm{PC})$ for $\mathrm{Cu} \mid \mathrm{Li}$, and $\mathrm{Li} \mid \mathrm{Li}$ cells that were cycled at $1 \mathrm{mAh} \mathrm{cm}^{-2}$ was blended with $5 \mathrm{wt}-\%$ fluoroethylene carbonate. 
Characterization. A Rigaku powder X-ray diffractometer tested XRD. Fourier Transform Infrared Spectroscopy (FTIR) data were recorded on a Nicolet 6700 FTIR. Raman spectra were recorded on a Renishaw inVia Raman microscope spectrometer $(633 \mathrm{~nm})$. SEM images were taken using FEI Nova 230. Thermogravimetric analysis (TGA) was tested in $\mathrm{N}_{2}$ or air atmospheres (ramping rate: $10{ }^{\circ} \mathrm{C} \mathrm{min}^{-1}$ ) using TA instruments Q500. Mechanical tensile-stress experiments were carried out using an Instron 5942 at a strain rate of $5 \mathrm{~mm} \mathrm{~min}^{-1}$. The $\mathrm{N}_{2}$ isotherms of activated UiO-66 were obtained from Micromeritics ASAP 2020 PLUS. The surface area was calculated based on the Brunauer-Emmett-Teller theory. The corresponding fitting of pore size distribution employed a slit-pore geometry and 2D-NLDFT model with a standard deviation below 2 .

Electrochemical characterizations. The ionic conductivity test was measured in a coin cell with stainless steel (SS)|gel electrolyte|SS structure. Alternating current (AC) impedance spectroscopy was used, and the frequency ranged from $500 \mathrm{kHz}$ to $1 \mathrm{~Hz}$. A voltage amplitude of $10 \mathrm{mV}$ was applied. The cells were tested from room temperature to $80{ }^{\circ} \mathrm{C}$. The conductivity $\sigma$ was calculated according to the equation:

$$
\sigma=\frac{L}{R A}
$$

$\mathrm{L}(\mathrm{cm})$ is the thickness of the gel, $\mathrm{R}(\Omega)$ is the resistance tested by AC impedance spectroscopy, and $\mathrm{A}\left(\mathrm{cm}^{2}\right)$ represents the area of SS disc.

Potentiostatic polarization method was used to obtain the $\mathrm{Li}^{+}$transference number based on the following equation:

$$
\mathrm{t}_{\mathrm{Li}^{+}}=\frac{I_{S}\left(\Delta V-I_{0} R_{0}\right)}{I_{0}\left(\Delta V-I_{S} R_{S}\right)}
$$

$\Delta \mathrm{V}$ is the voltage polarization, and the applied voltage is $20 \mathrm{mV}, \mathrm{I}_{0}$ and $\mathrm{I}_{\mathrm{s}}$ are the initial and steadystate current, and $\mathrm{R}_{0}$ and $\mathrm{R}_{\mathrm{s}}$ are the initial and steady-state resistance, respectively.

Electrode material $\left(\mathrm{LiFePO}_{4} / \mathrm{LiNi}_{0.6} \mathrm{Co}_{0.2} \mathrm{Mn}_{0.2} \mathrm{O}_{2} / \mathrm{Li}_{4} \mathrm{Ti}_{5} \mathrm{O}_{12}\right)$ was mixed with conductive carbon and polyvinylidene difluoride (PVDF) in a mass ratio of 80:10:10. The solvent N-methyl2-pyrrolidone (NMP) was added into the solid powder to obtain a uniform slurry. The slurry was cast on $\mathrm{Al}$ foil and dried at $90{ }^{\circ} \mathrm{C}$ under vacuum overnight. The Al foil coated with cathode material was tailored into discs (diameter $14 \mathrm{~mm}$ ). The mass loading of the cathode was ca. $6 \mathrm{mg} \mathrm{cm}^{-2}$. The coin cells were assembled in a glove box without adding liquid electrolytes. 


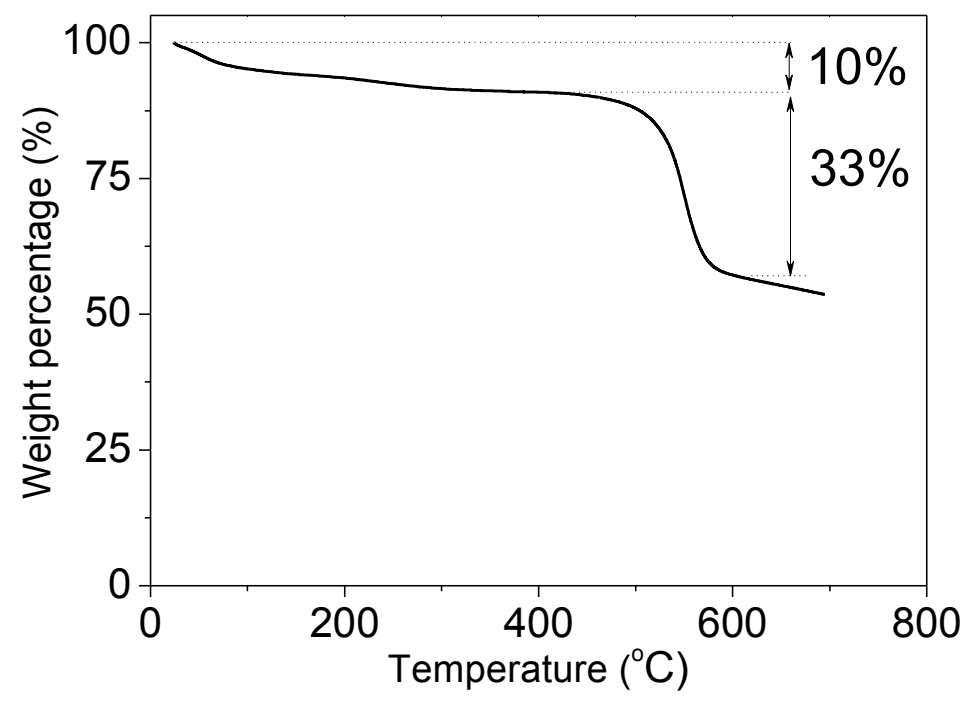

Figure S1. TG curve of UiO-66 in $\mathrm{N}_{2}$ atmosphere.

Table S1. Mechanical performance of GPL and MGPLs.

\begin{tabular}{cccc}
\hline Sample & Stress (MPa) & Strain (\%) & Modulus (MPa) \\
\hline GPL & $1.23 \pm 0.35$ & $138.7 \pm 21.2$ & $2.03 \pm 0.6$ \\
MGPL-12 & $1.09 \pm 0.46$ & $101.3 \pm 28.7$ & $2.03 \pm 0.25$ \\
MGPL-24 & $0.39 \pm 0.04$ & $92.4 \pm 2.8$ & $0.66 \pm 0.09$ \\
MGPL-50 & $0.27 \pm 0.03$ & $76.3 \pm 8.3$ & $0.46 \pm 0.04$ \\
\hline
\end{tabular}

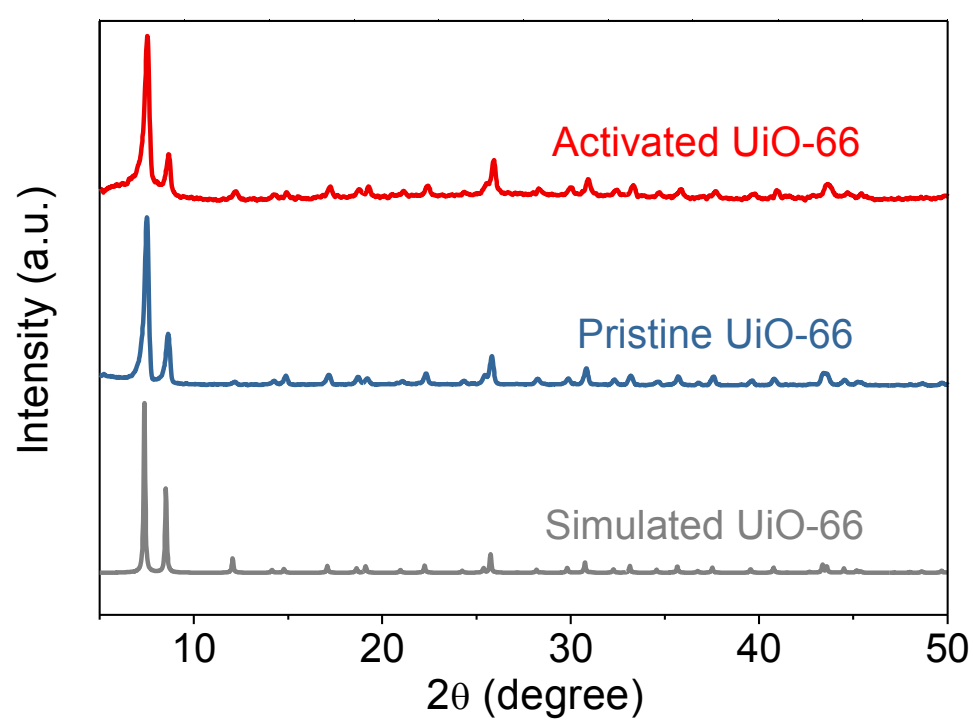

Figure S2. XRD patterns of simulated, pristine, and activated UiO-66. 


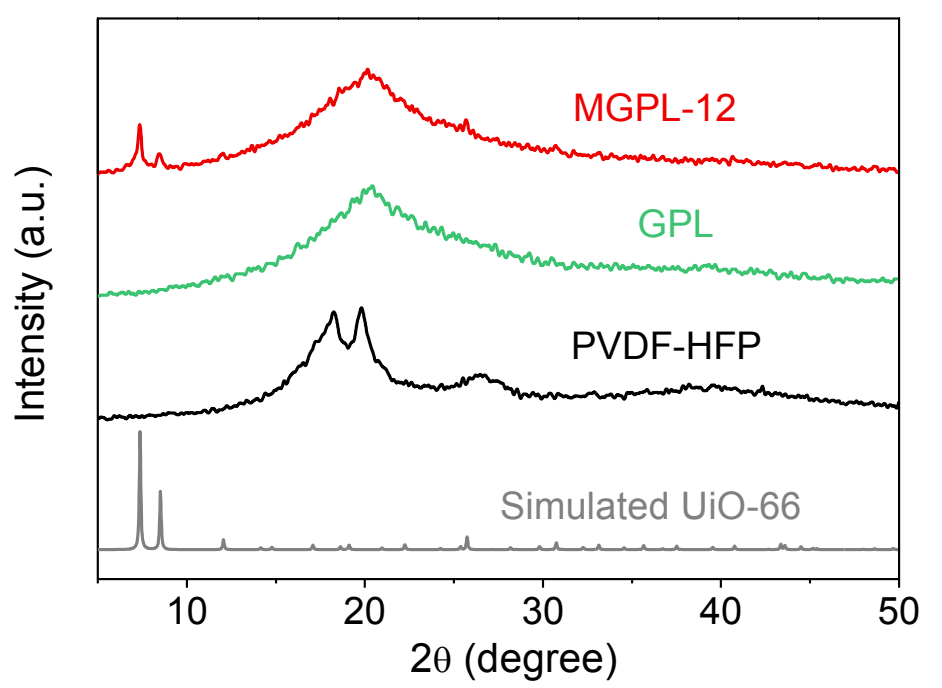

Figure S3. XRD patterns of simulated UiO-66, PVDF-HFP powders, GPL, and MGPL-12.

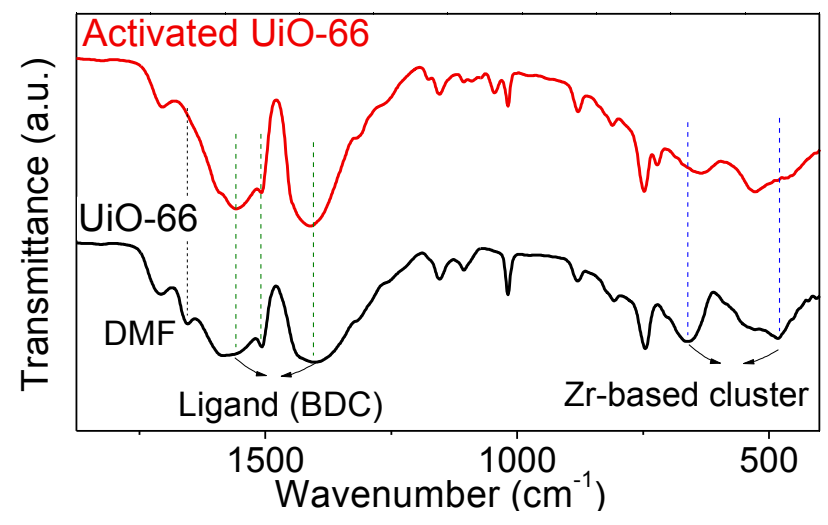

Figure S4. IR spectra of UiO-66 and activated UiO-66.

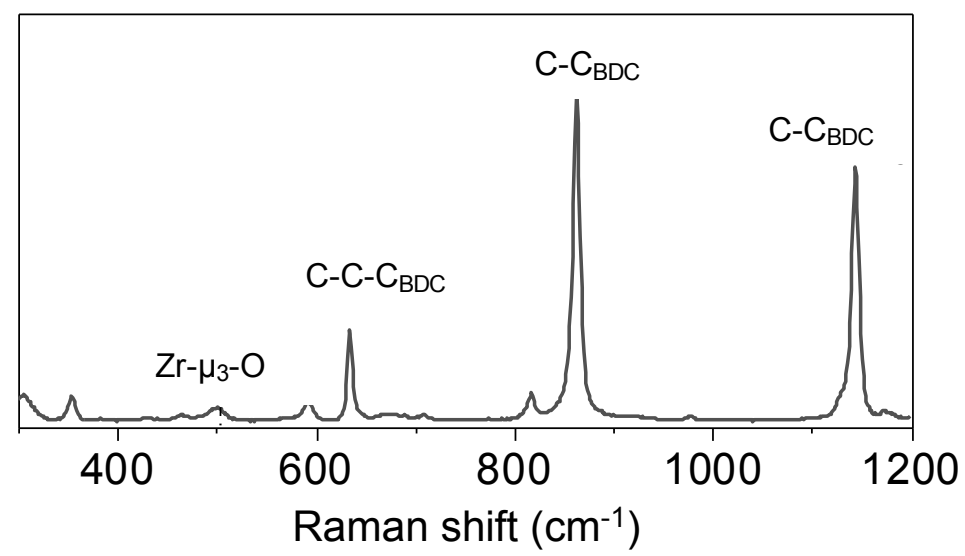

Figure S5. Raman spectra of activated UiO-66. 


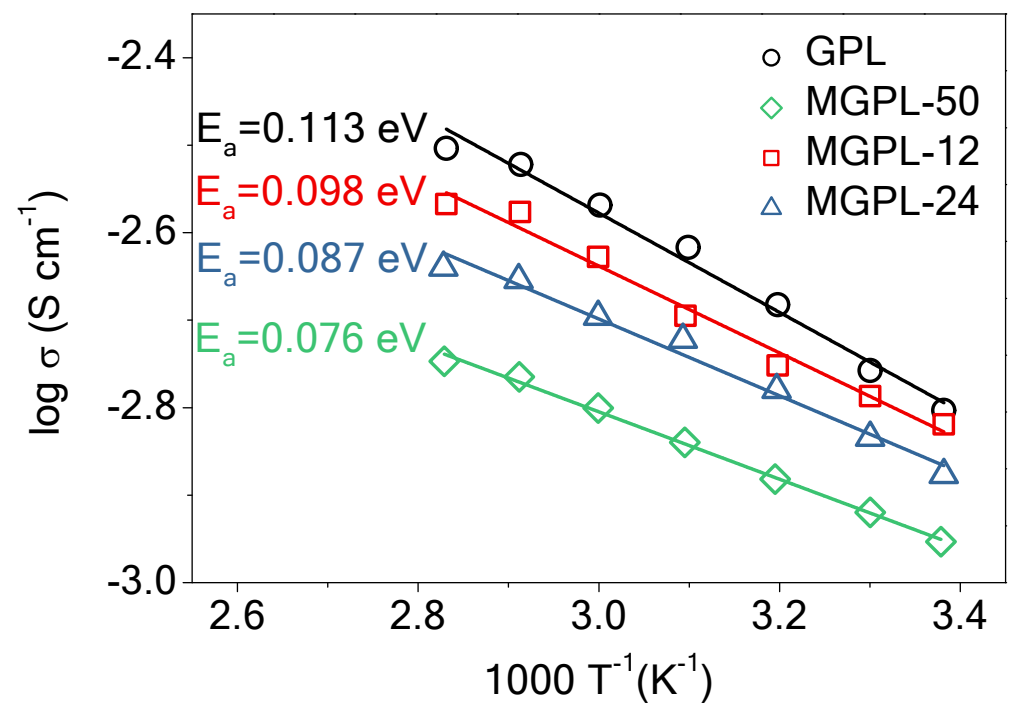

Figure S6. Temperature-dependent ionic conductivities of electrolyte membranes (GPL and MGPLs) and linear fittings based on the Arrhenius relationship.
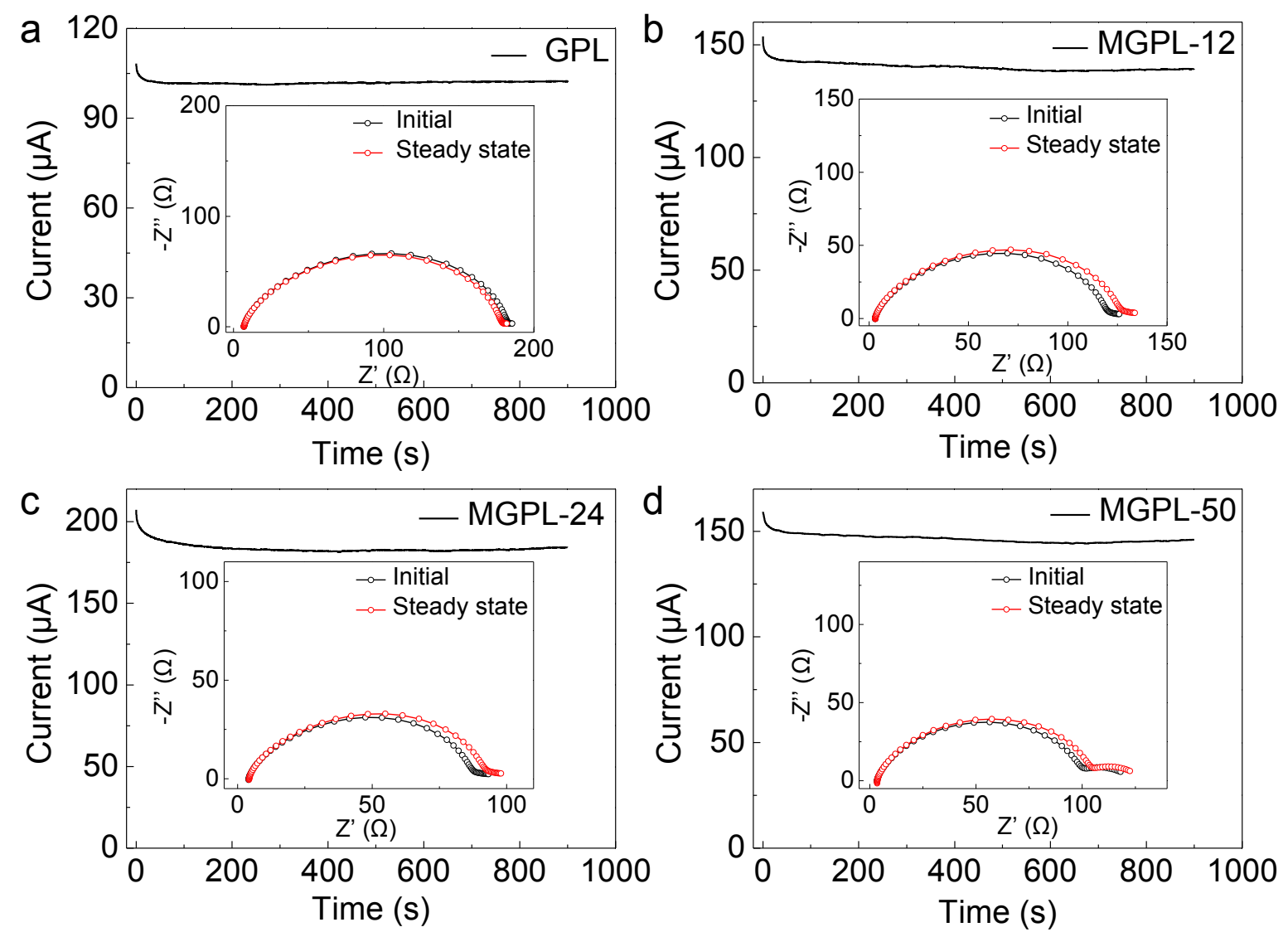

Figure S7. Measurements of $\mathrm{t}_{\mathrm{Li}}{ }^{+}$based on a potentiostat approach. 
Table S2. Conductivity and $\mathrm{Li}^{+}$transference number comparisons between polymer electrolytes with various filler materials

\begin{tabular}{|c|c|c|c|c|}
\hline Electrolyte system & $\begin{array}{l}\text { Conductivity } \\
\qquad\left(\mathrm{S} \mathrm{cm}^{-1}\right)\end{array}$ & $\begin{array}{l}\text { Temperature } \\
\left({ }^{\circ} \mathrm{C}\right)\end{array}$ & $\mathrm{t}_{\mathrm{Li}^{+}}$ & Ref. \\
\hline MGPL-12 & $1.52 \times 10^{-3}$ & 25 & 0.64 & $\begin{array}{l}\text { This } \\
\text { work }\end{array}$ \\
\hline [EMIM][TFSI]-LiTFSI-(MOF-525) & $3 \times 10^{-4}$ & 25 & 0.36 & 1 \\
\hline PEO-LiTFSI-PC-SiO ${ }_{2}$ & $5 \times 10^{-3}$ & 25 & $\mathrm{n} / \mathrm{a}$ & 2 \\
\hline PEO-LiTFSI-LLTO & $5.53 \times 10^{-5}$ & 25 & 0.195 & 3 \\
\hline $\mathrm{PAN} \mathrm{LiClO}_{4}$-LLTO & $2.4 \times 10^{-4}$ & 25 & $\mathrm{n} / \mathrm{a}$ & 4 \\
\hline LiTFSI-[Py $\left.{ }_{13}\right][\mathrm{TFSI}]-\mathrm{SiO}_{2}$ & $1.37 \times 10^{-3}$ & 30 & 0.22 & 5 \\
\hline PEO-LiTFSI-LLZO & $2.5 \times 10^{-4}$ & 25 & $\mathrm{n} / \mathrm{a}$ & 6 \\
\hline PEO-LiTFSI-LLZTO & $1.12 \times 10^{-5}$ & 25 & 0.58 & 7 \\
\hline $\mathrm{LiClO}_{4}$-PVDF-LLZTO & $5 \times 10^{-4}$ & 25 & $\mathrm{n} / \mathrm{a}$ & 8 \\
\hline $\begin{array}{c}\text { PEO-LiTFSI-Cationic }\left(\mathrm{UiO}-66-\mathrm{NH}_{2}\right) \\
(12.5 \text { vol- } \%)\end{array}$ & $3.1 \times 10^{-5}$ & 25 & 0.72 & 9 \\
\hline $\begin{array}{l}\text { PEO-LiTFSI-(Mg-BTC) } \\
\qquad(10 \mathrm{wt}-\%)\end{array}$ & $2 \times 10^{-4}$ & 30 & 0.4 & 10 \\
\hline $\begin{array}{l}\text { PEO-LiTFSI-(MOF-5) } \\
(10 \mathrm{wt}-\%)\end{array}$ & $3.16 \times 10^{-5}$ & 25 & $\mathrm{n} / \mathrm{a}$ & 11 \\
\hline $\begin{array}{l}\text { PVDF- LiTFSI-(UiO-LiTFSI) } \\
\text { (56 wt-\%) }\end{array}$ & $2.07 \times 10^{-4}$ & 25 & 0.84 & 12 \\
\hline $\begin{array}{l}\text { PEO-LiTFSI-(Al-BTC) } \\
\qquad(10 \mathrm{wt}-\%)\end{array}$ & $\sim 1 \times 10^{-5}$ & 30 & 0.55 & 13 \\
\hline PEO-(BMIM-PF $)-(\mathrm{Cu}-\mathrm{BTC})$ & $5.3 \times 10^{-4}$ & 25 & $\mathrm{n} / \mathrm{a}$ & 14 \\
\hline PEO-LiTFSI-(Al-MOF) (5 wt-\%) & $2.09 \times 10^{-5}$ & 30 & 0.46 & 15 \\
\hline PEO-LiTFSI-(MIL-53) (10 wt-\%) & $1.62 \times 10^{-5}$ & 30 & 0.34 & 16 \\
\hline
\end{tabular}




\begin{tabular}{|c|c|c|c|c|}
\hline PEO-LiTFSI-(Ni-BTC) $(10 \mathrm{wt}-\%)$ & $1.4 \times 10^{-4}$ & 30 & 0.4 & 17 \\
\hline PEO-LiTFSI-(Cu-BDC) $(10 \mathrm{wt} \%)$ & $9 \times 10^{-5}$ & 30 & 0.41 & 18 \\
\hline PVDF-(MOF-74) & $6.72 \times 10^{-4}$ & 25 & 0.66 & 19 \\
\hline PEO-LiPF $-(\mathrm{UiO}-66)$ & $1.47 \times 10^{-4}$ & 30 & 0.47 & 20 \\
\hline$[$ EMIM][TFSI]-PEO-(UiO-66) & $1.3 \times 10^{-4}$ & 30 & 0.35 & 21 \\
\hline
\end{tabular}

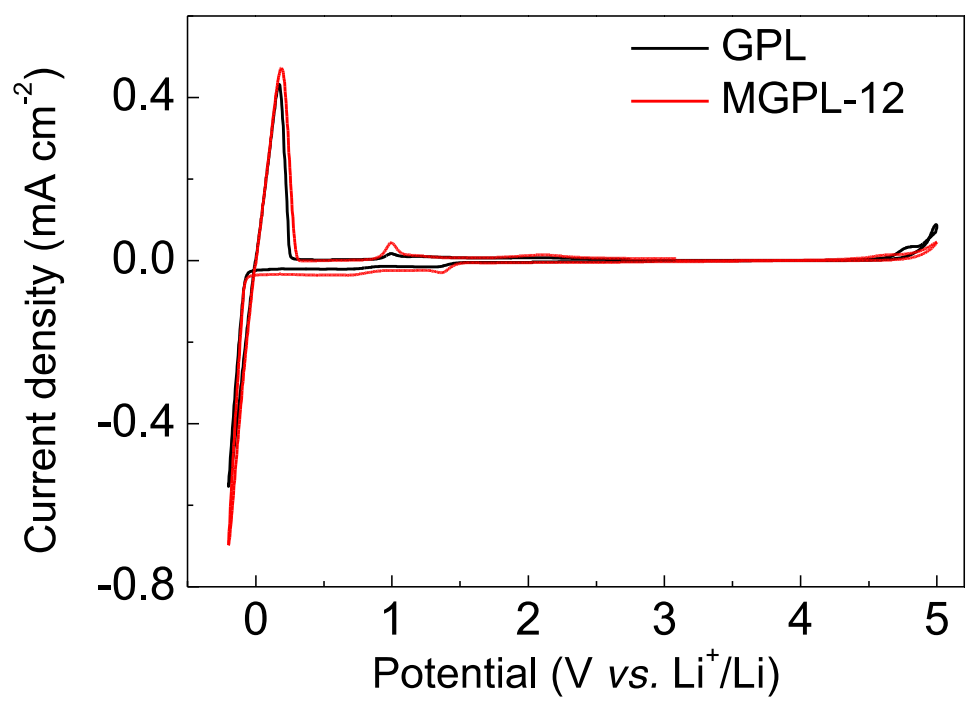

Figure S8. CV curves of GPL and MGPL-12 sandwiched by Li metal and stainless-steel electrodes.
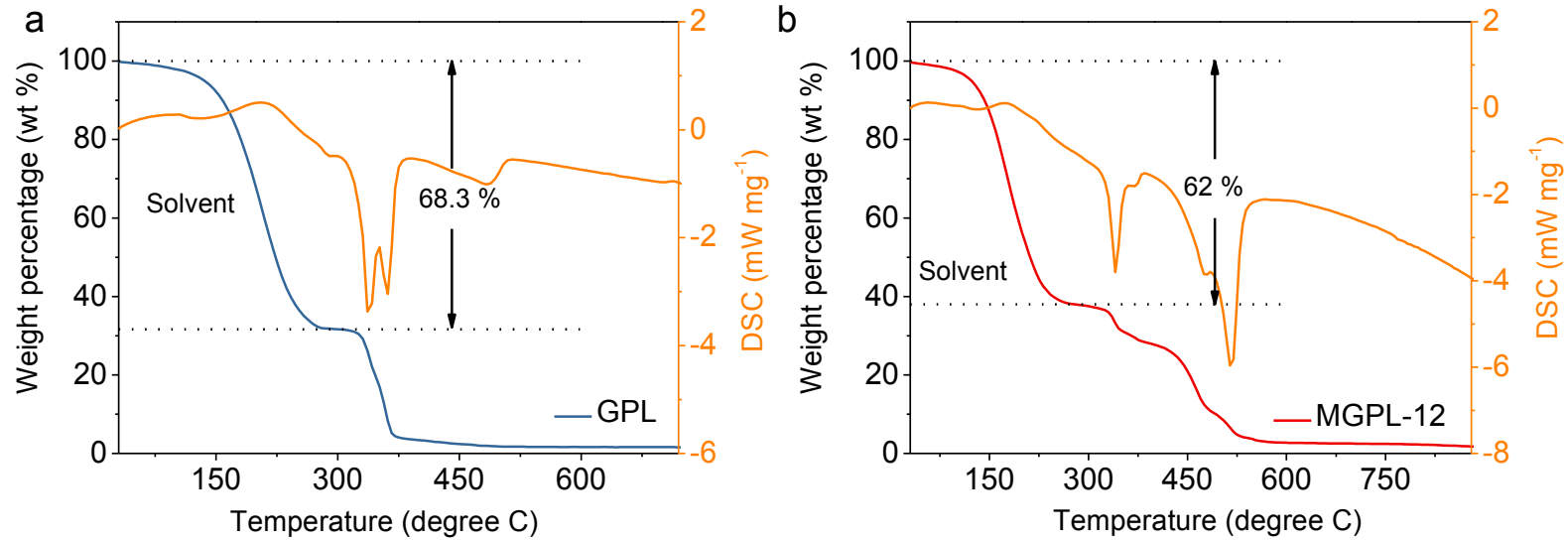

Figure S9. TG curves of GPL and MGPL-12 in the air atmosphere. 


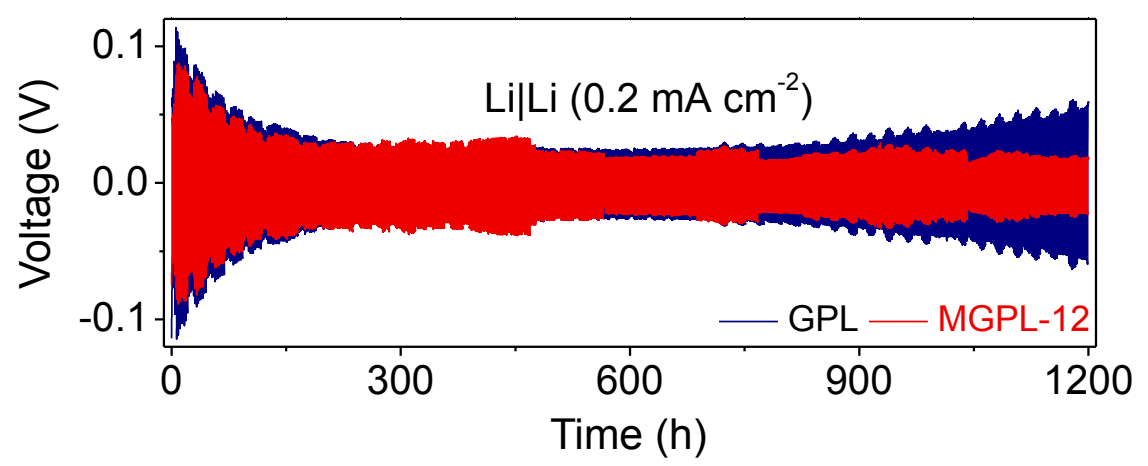

Figure S10. Polarization tests of Li|Li cell under $0.2 \mathrm{~mA} \mathrm{~cm} \mathrm{~cm}^{-2}$ using GPL and MGPL-12.
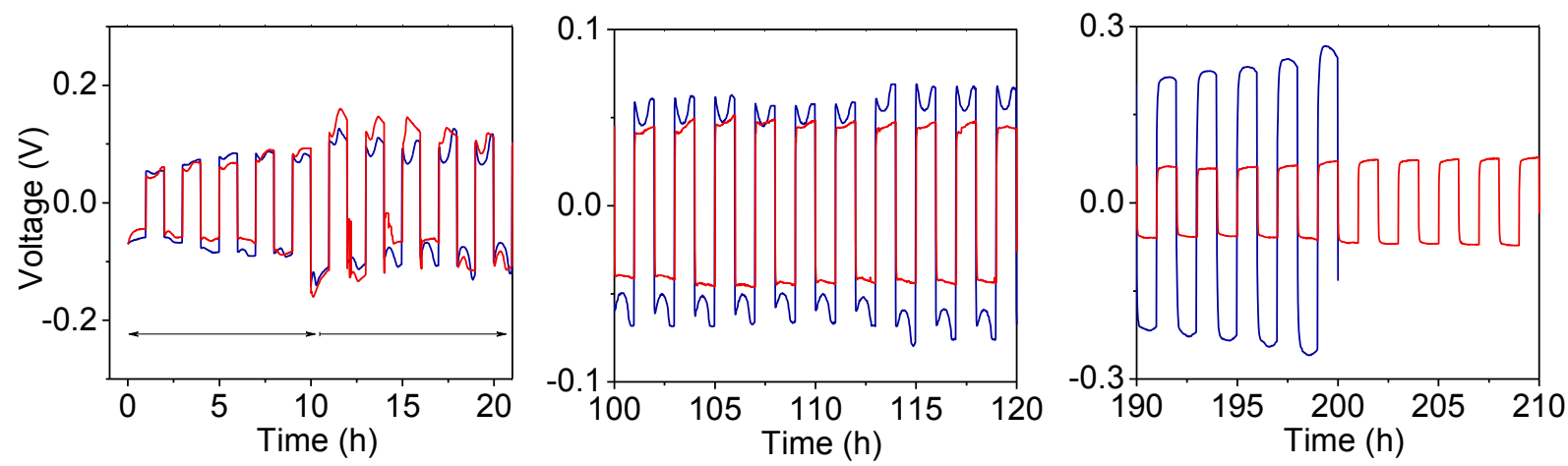

Figure S11. Polarization tests of $\mathrm{Li} \mid \mathrm{Li}$ cells at $0.2 \mathrm{~mA} \mathrm{~cm}{ }^{-2}$ (first $10 \mathrm{~h}$ ) and $0.5 \mathrm{~mA} \mathrm{~cm}-2$ (subsequent $200 \mathrm{~h}$ ) with a time interval of $1 \mathrm{~h}$.

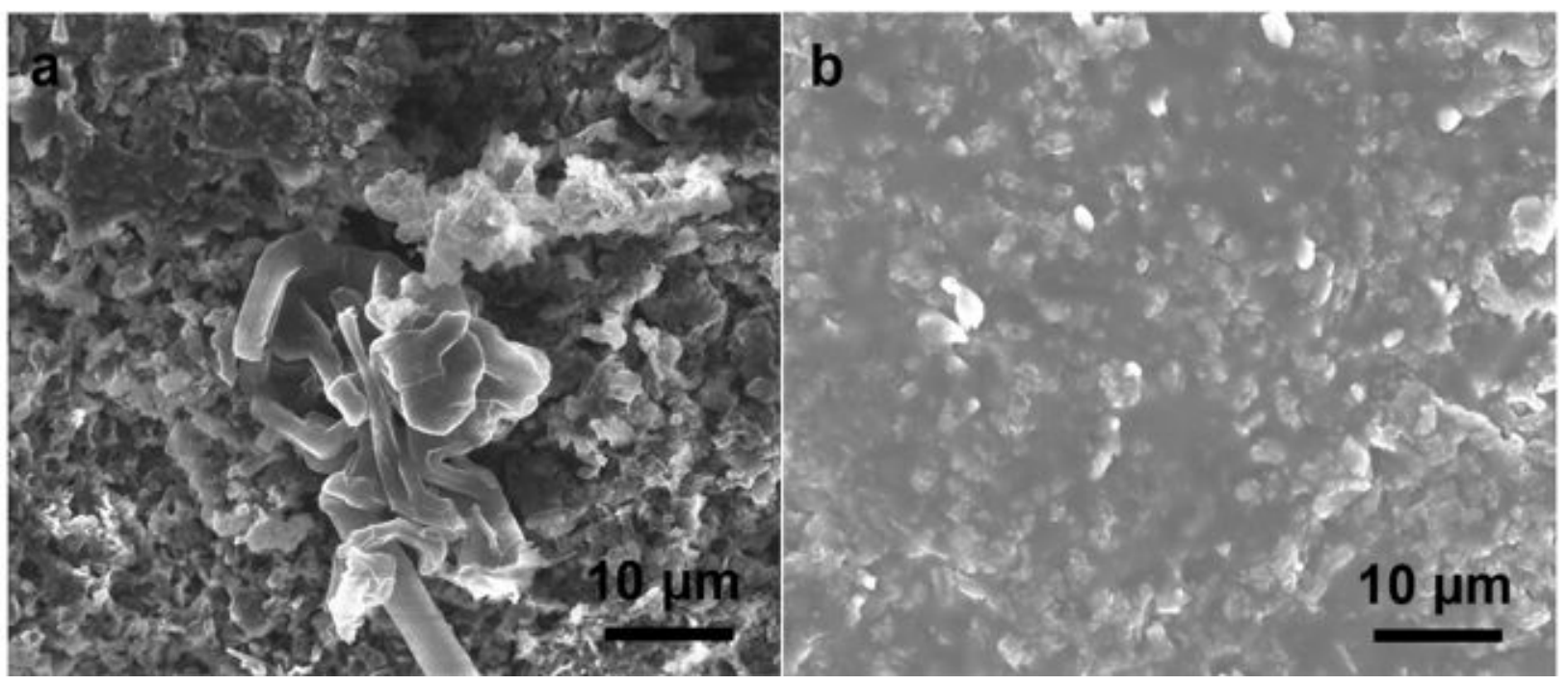

Figure S12. SEM images of Li metal surfaces harvested from cycled Li|Li cells (100 cycles, 0.5 $\mathrm{mA} \mathrm{cm}{ }^{-2}$ ) using (a) GPL and (b) MGPL-12. 


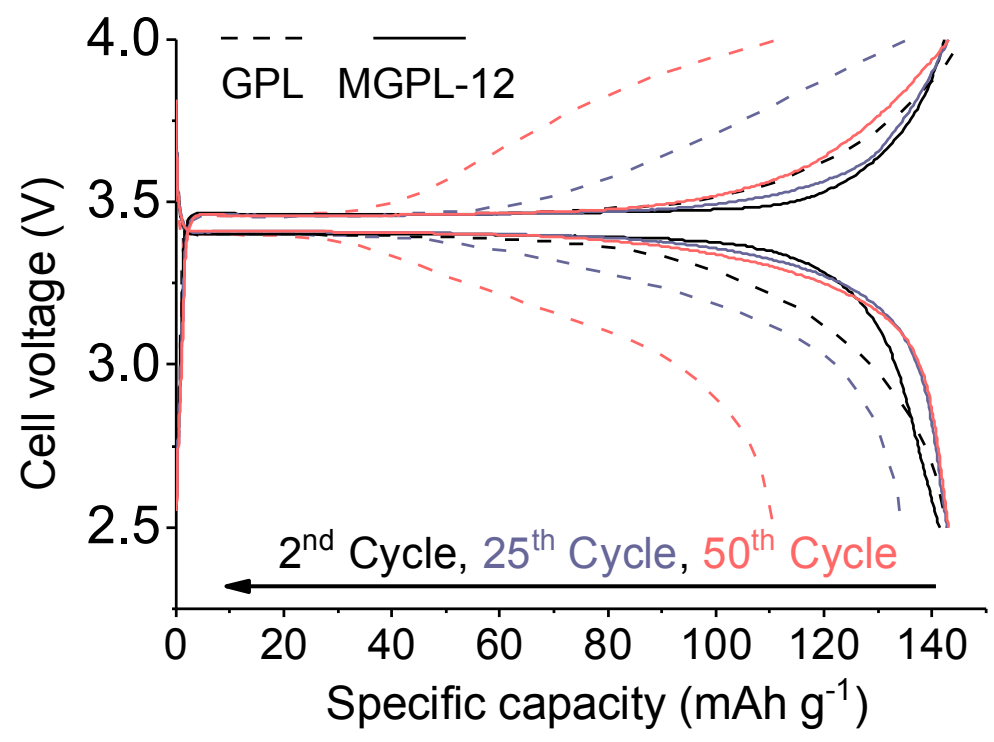

Figure S13. Galvanostatic charge/discharge curves of $\mathrm{LiFePO}_{4} \mid \mathrm{Li}$ cells $(0.2 \mathrm{C})$
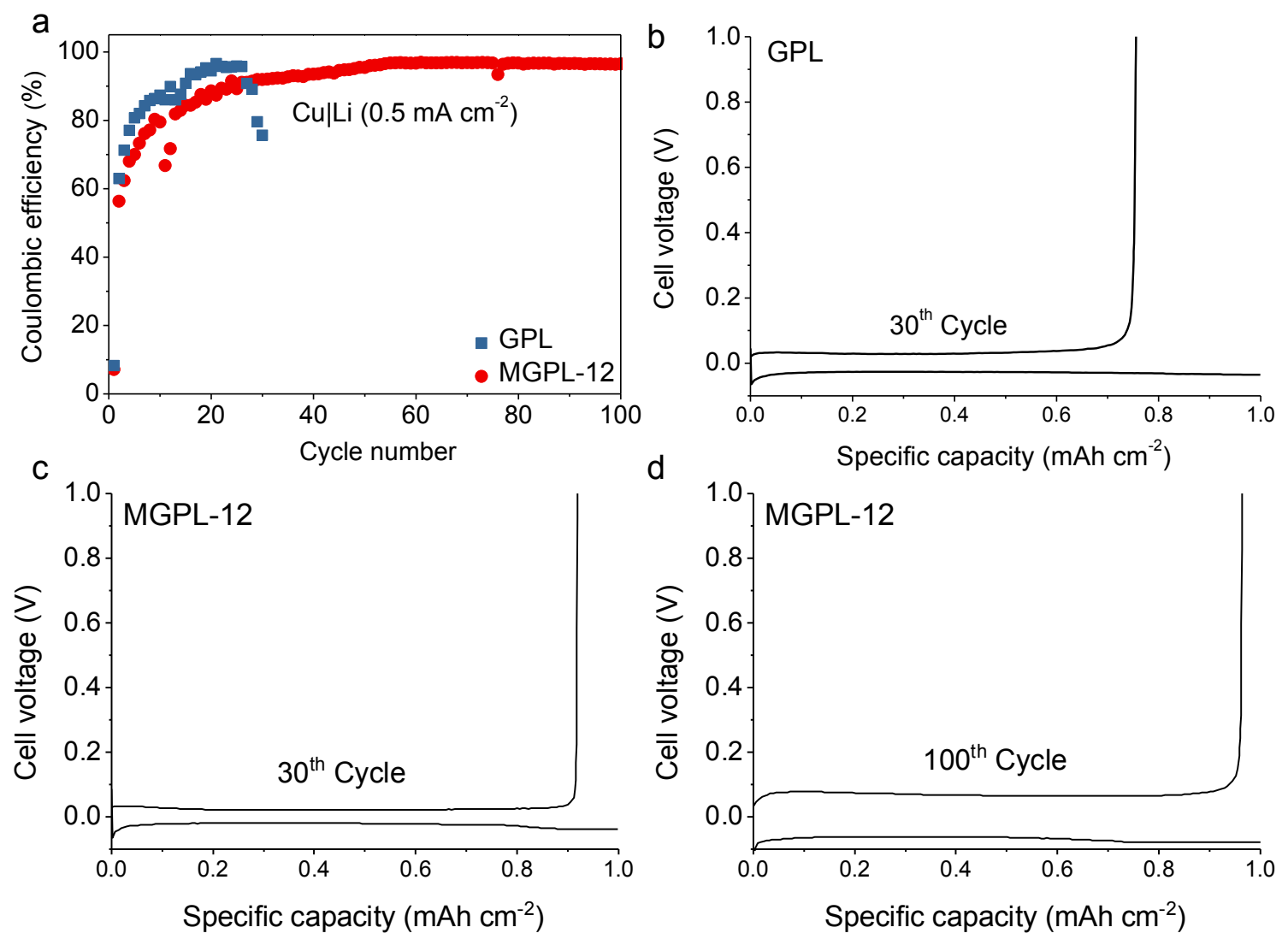

Figure S14. (a) Coulombic efficiency of $\mathrm{Cu} / \mathrm{Li}$ cell at a current density of $0.5 \mathrm{~mA} \mathrm{~cm} \mathrm{c}^{-2}$ and deposition capacity of $1 \mathrm{mAh} \mathrm{cm}^{-2}$ (first 10 cycles were activated at $0.05 \mathrm{~mA} \mathrm{~cm}^{-2}$ ). (b) Voltage profile of $\mathrm{Cu} \mid \mathrm{Li}$ cell using GPL in the $30^{\text {th }}$ cycle. Voltage profiles of $\mathrm{Cu} \mid \mathrm{Li}$ cell using MGPL-12 at (c) $30^{\text {th }}$ cycle and (d) $100^{\text {th }}$ cycle. 


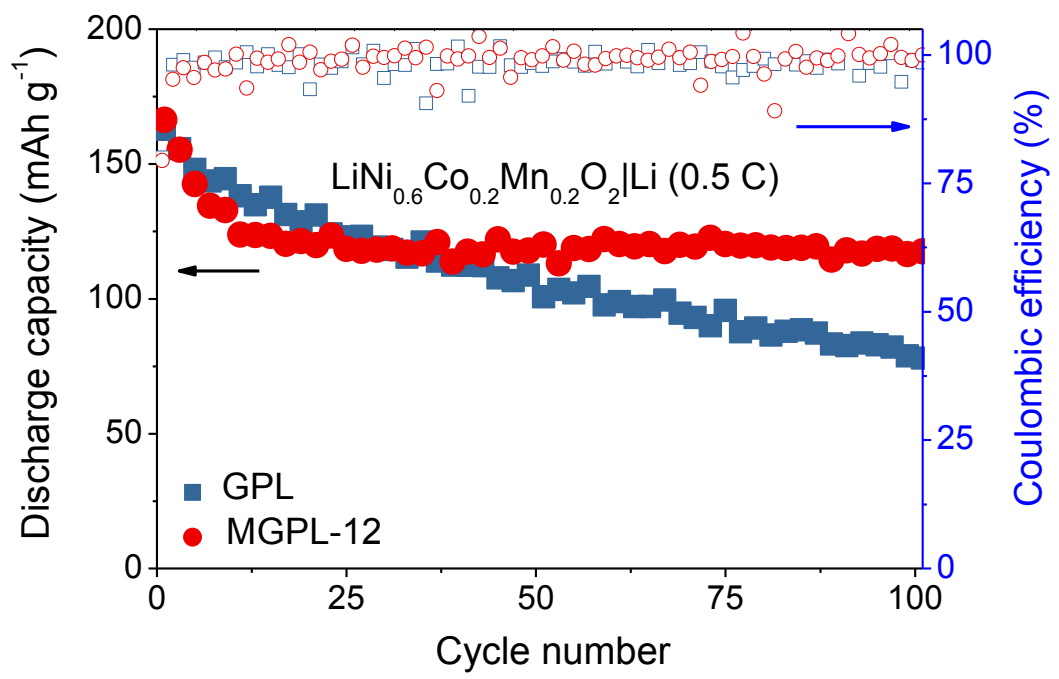

Figure S15. Galvanostatic cycling of $\mathrm{LiNi}_{0.6} \mathrm{Co}_{0.2} \mathrm{Mn}_{0.2} \mathrm{O}_{2} \mid \mathrm{Li}$ cells at $0.5 \mathrm{C}$ using GPL and MGPL12.
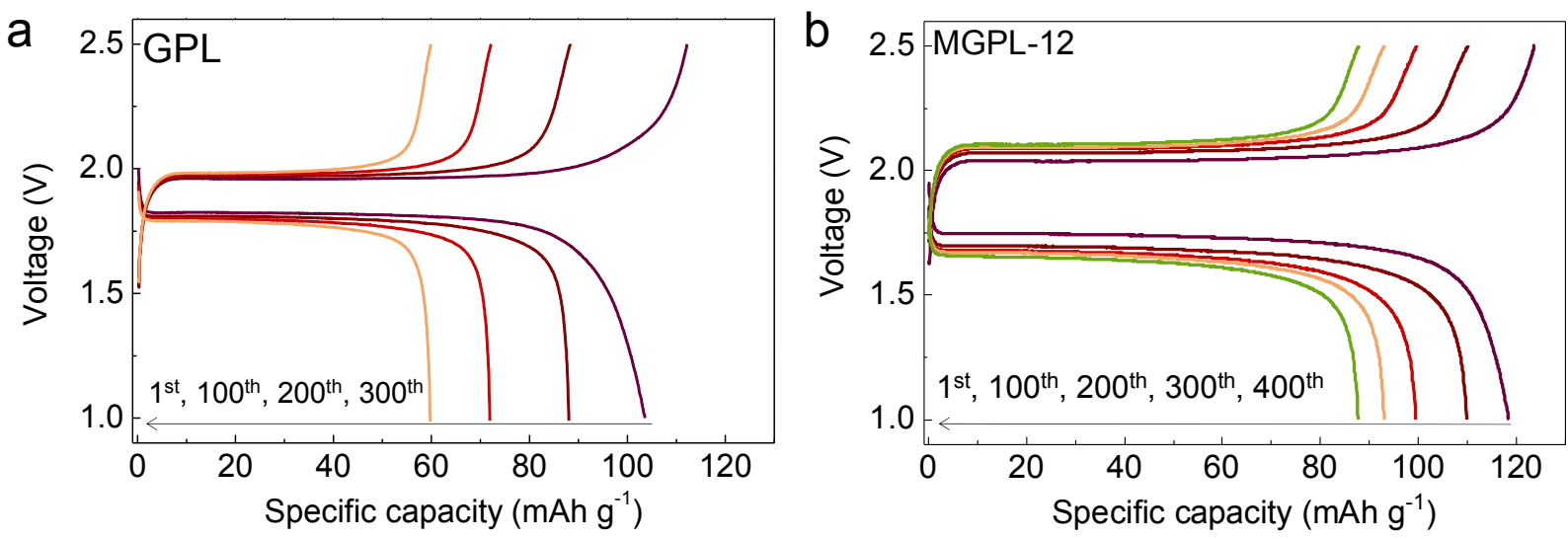

Figure S16. Galvanostatic charge/discharge curves of $\mathrm{LiFePO}_{4} \mid \mathrm{Li}_{4} \mathrm{Ti}_{5} \mathrm{O}_{12}$ full cells $(0.5 \mathrm{C})$ 


\section{Supporting references}

(1) Ziqi, W.; Rui, T.; Hongbin, W.; Luyi, Y.; Jiangtao, H.; Haibiao, C.; Feng, P., A MetalOrganic-Framework-Based Electrolyte with Nanowetted Interfaces for High-Energy-Density Solid-State Lithium Battery. Adv. Mater. 2018, 30 (2), 1704436.

(2) Choudhury, S.; Mangal, R.; Agrawal, A.; Archer, L. A., A highly reversible room-temperature lithium metal battery based on crosslinked hairy nanoparticles. Nat. Commun. 2015, 6 .

(3) Zhu, L.; Zhu, P.; Fang, Q.; Jing, M.; Shen, X.; Yang, L., A novel solid PEO/LLTO-nanowires polymer composite electrolyte for solid-state lithium-ion battery. Electrochim. Acta 2018, 292, 718-726.

(4) Liu, W.; Liu, N.; Sun, J.; Hsu, P.-C.; Li, Y.; Lee, H.-W.; Cui, Y., Ionic Conductivity Enhancement of Polymer Electrolytes with Ceramic Nanowire Fillers. Nano Lett. 2015, 15 (4), 2740-2745.

(5) Li, L.; Yang, X.; Li, J.; Xu, Y., A novel and shortcut method to prepare ionic liquid gel polymer electrolyte membranes for lithium-ion battery. Ionics 2018, 24 (3), 735-741.

(6) Fu, K.; Gong, Y. H.; Dai, J. Q.; Gong, A.; Han, X. G.; Yao, Y. G.; Wang, C. W.; Wang, Y. B.; Chen, Y. N.; Yan, C. Y.; Li, Y. J.; Wachsman, E. D.; Hu, L. B., Flexible, solid-state, ion-conducting membrane with 3D garnet nanofiber networks for lithium batteries. Proc. Natl. Acad. Sci. U. S. A. 2016, 113 (26), 7094-7099.

(7) Zhao, C.-Z.; Zhang, X.-Q.; Cheng, X.-B.; Zhang, R.; Xu, R.; Chen, P.-Y.; Peng, H.-J.; Huang, J.-Q.; Zhang, Q., An anion-immobilized composite electrolyte for dendrite-free lithium metal anodes. Proc. Natl. Acad. Sci. U. S. A. 2017, 201708489.

(8) Zhang, X.; Liu, T.; Zhang, S.; Huang, X.; Xu, B.; Lin, Y.; Xu, B.; Li, L.; Nan, C.-W.; Shen, Y., Synergistic Coupling Between Li6. 75La3Zr1. 75Ta0. 25O12 and Poly (vinylidene fluoride) Induces High Ionic Conductivity, Mechanical Strength and Thermal Stability of Solid Composite Electrolytes. J. Am. Chem. Soc. 2017.

(9) Huo, H.; Wu, B.; Zhang, T.; Zheng, X.; Ge, L.; Xu, T.; Guo, X.; Sun, X., Anion-immobilized polymer electrolyte achieved by cationic metal-organic framework filler for dendrite-free solid-state batteries. Energy Storage Materials 2019, 18, 59-67.

(10) Angulakshmi, N.; Kumar, R. S.; Kulandainathan, M. A.; Stephan, A. M., Composite Polymer Electrolytes Encompassing Metal Organic Frame Works: A New Strategy for All-Solid-State Lithium Batteries. The Journal of Physical Chemistry C 2014, 118 (42), 24240-24247.

(11) Yuan, C.; Li, J.; Han, P.; Lai, Y.; Zhang, Z.; Liu, J., Enhanced electrochemical performance of poly(ethylene oxide) based composite polymer electrolyte by incorporation of nano-sized metal-organic framework. J. Power Sources 2013, 240, 653-658.

(12) Zhu, F.; Bao, H.; Wu, X.; Tao, Y.; Qin, C.; Su, Z.; Kang, Z., High-Performance Metal-Organic FrameworkBased Single Ion Conducting Solid-State Electrolytes for Low-Temperature Lithium Metal Batteries. ACS Appl. Mater. Interfaces 2019, 11 (46), 43206-43213.

(13) Gerbaldi, C.; Nair, J. R.; Kulandainathan, M. A.; Kumar, R. S.; Ferrara, C.; Mustarelli, P.; Stephan, A. M., Innovative high performing metal organic framework (MOF)-laden nanocomposite polymer electrolytes for all-solidstate lithium batteries. Journal of Materials Chemistry A 2014, 2 (26), 9948-9954.

(14) Dutta, R.; Kumar, A., Ion transport dynamics in ionic liquid incorporated CuBTC-metal-organic framework based composite polymer electrolyte. Journal of Materials Science: Materials in Electronics 2019, 30 (2), 1117-1132.

(15) Zhang, Z.; You, J.-H.; Zhang, S.-J.; Wang, C.-W.; Zhou, Y.; Li, J.-T.; Huang, L.; Sun, S.-G., Metal Organic Framework Nanorod Doped Solid Polymer Electrolyte with Decreased Crystallinity for High-Performance All-SolidState Lithium Batteries. ChemElectroChem 2020, 7 (5), 1125-1134.

(16) Zhu, K.; Liu, Y.; Liu, J., A fast charging/discharging all-solid-state lithium ion battery based on PEO-MIL53(Al)-LiTFSI thin film electrolyte. RSC Advances 2014, 4 (80), 42278-42284.

(17) Suriyakumar, S.; Kanagaraj, M.; Angulakshmi, N.; Kathiresan, M.; Nahm, K. S.; Walkowiak, M.; Wasiński, 
K.; Półrolniczak, P.; Stephan, A. M., Charge-discharge studies of all-solid-state Li/LiFePO4 cells with PEO-based composite electrolytes encompassing metal organic frameworks. RSC Advances 2016, 6 (99), 97180-97186.

(18) Senthil Kumar, R.; Raja, M.; Anbu Kulandainathan, M.; Manuel Stephan, A., Metal organic framework-laden composite polymer electrolytes for efficient and durable all-solid-state-lithium batteries. RSC Advances 2014, 4 (50), 26171-26175.

(19) Han, D.-D.; Wang, Z.-Y.; Pan, G.-L.; Gao, X.-P., Metal-Organic-Framework-Based Gel Polymer Electrolyte with Immobilized Anions To Stabilize a Lithium Anode for a Quasi-Solid-State Lithium-Sulfur Battery. ACS Appl. Mater. Interfaces 2019, 11 (20), 18427-18435.

(20) Zhang, Z.; Huang, Y.; Gao, H.; Hang, J.; Li, C.; Liu, P., MOF-derived ionic conductor enhancing polymer electrolytes with superior electrochemical performances for all solid lithium metal batteries. Journal of Membrane Science 2020, 598, 117800.

(21) Wu, J.-F.; Guo, X., MOF-derived nanoporous multifunctional fillers enhancing the performances of polymer electrolytes for solid-state lithium batteries. J. Mater. Chem. A 2019, 7 (6), 2653-2659. 